\title{
La importancia de la cuarta edición en relación con los diminutivos que aparecen en el DRAE
}

\author{
Rafael García Pérez \\ Universidad de Salamanca
}

1. Quien consulte con alguna atención el diccionario académico, se sorprenderá, sin duda, por la gran cantidad de diminutivos que acoge. Alrededor de un 1,87\% de los lemas llevan alguna de sus marcas, si tenemos en cuenta tanto la parte central de la definición como la parte etimológica, y alrededor de un $1 \%$ si nos centramos exclusivamente en la primera'.

Este grupo de palabras, ya notable en el Diccionario de Autoridades, se ha ido ampliando a lo largo de sus diversas ediciones ${ }^{2}$. Sin em-

1 La mera presencia de una abreviatura no significa que el diccionario considere a la palabra actualizable como diminutivo, porque un vocablo creado con este valor puede haberse lexicalizado y haber perdido su sentido original, o bien proceder de un diminutivo en una lengua extranjera y no usarse como tal en español. En estos casos, el diccionario ha optado por recoger la marca en la etimología, es decir, entre paréntesis inmediatamente después de la entrada. Por el contrario, para indicar que el valor diminutivo está presente, ha optado por colocar la marca en la definición, siempre como primera acepción o primera subacepción. Hay que señalar también que se puede referir a un derivado en la lengua de hoy, lo que implica la ausencia de marcas añadidas, o a un derivado ya obsoleto, para lo cual presenta marcas que acompañan a la abreviatura y que determinan su carácter de arcaísmo. Tomemos como ejemplo polluelo y herbecica. "Polluelo, la. (De pollo') m. y f. d. de pollo". "herbecica, ta. f. d. ant. de hierba". Sobre el problema de los arcaísmos en el diccionario y, especialmente en el DRAE, v. Pascual (1997).

2 El incremento ha sido progresivo, aun cuando hayamos de contar con un pequeño porcentaje que se ha visto suprimido en algunas de las ediciones: la duodécima, por ejemplo. 
bargo, hay una edición particularmente importante: la cuarta (1803), que es la que presenta de hecho el mayor número de nuevos diminutivos y que condiciona, por ello, la macroestructura del actual diccionario académico ${ }^{3}$.

Si consultamos los datos recogidos en el apéndice, comprobaremos que, entre la primera y la tercera edición, las palabras que se introducen pertenecen casi exclusivamente a las seis letras iniciales del abecedario, es decir, aquéllas comprendidas entre la $A$ y la $F^{4}$. Pero, mientras la primera y la segunda edición se detienen sobre todo en la letra $C^{5}$, la tercera admite novedades en las seis.

Sabemos que, en el momento de plantearse la reedición del Diccionario de Autoridades, convenientemente corregido y aumentado, los académicos habían revisado únicamente las letras $A, B$, y $C$. Como el trabajo de revisión no estaba completo y no podía paralizarse, se encargó a una comisión la labor de confeccionar, para una fecha cercana, la nueva edición, que habría de aparecer en un solo tomo; pero esta comisión, a causa del apremio que sufrió, se limitó a introducir los cambios de las cédulas de corrección y aumento de autoridades existentes hasta entonces, dejando el resto prácticamente inalterado ${ }^{6}$. He aquí el motivo de por qué en la primera y en la segunda edición encontramos casi todas las novedades en las tres letras iniciales. En el prólogo a la primera edición podemos leer:

3 Hemos añadido un apéndice con las listas de diminutivos que ha ido recibiendo el diccionario desde 1780 .

4 Hasta la cuarta edición, momento en que también se lleva a cabo una importante reforma ortográfica, las palabras que comenzaban por $C h$ se incluían dentro de la letra $C$.

5 No hay que olvidar que la segunda edición se limita a incorporar las adiciones y reformas que aparecían en el suplemento de la anterior.

6 "La norma general establecida era muy clara: para las letras A y B, reproducir el texto corregido y aumentado del tomo I, tal como se había publicado en 1770; para la letra $\mathrm{C}$, tomar el original revisado, aún inédito, del tomo II; y para las restantes letras, hasta el final, copiar el texto de los tomos III al VI del diccionario de Autoridades" (Seco 1991). 
Como la Academia trabaja siempre en dar al suyo [Diccionario] toda la perfección y aumento que puede, nunca deja de recoger voces con que enriquecerle, aun de aquellas letras que se han publicado ya. Por esto sin embargo del considerable aumento que se dio al tomo primero en la segunda impresión, después de publicado se han recogido otras voces pertenecientes a la $\mathrm{A}$ y la $\mathrm{B}$, y siendo ya un número competente, pues llegan a unas mil y quinientas, ha parecido conveniente ponerlas al fin de este tomo por vía de suplemento, no habiéndose podido intercalar en sus lugares correspondientes, porque cuando se acabaron de examinar y ordenar, estaba ya impresa la A y la B.

Posteriormente se mencionan el resto de las letras:

La D y demás letras restantes van sin aumento ni corrección, como están en el diccionario antiguo; pero alterada su ortografía y conforme a las últimas reglas que ha establecido la Academia.

El prólogo a la segunda edición es, prácticamente, un calco de su precedente, pero se recoge una nota a pie de página donde se señala que se ha tenido en cuenta el suplemento anterior y que se ha creado otro por si se llegase a imprimir una nueva edición. Ahora bien, este suplemento abarca otra vez las letras $A, B$ y $C$. Según lo que hemos visto, de la $D$ en adelante no se habría producido ningún cambio o, más bien, casi ninguno. Frente a las palabras del texto preliminar, los datos nos muestran que en estas ediciones se añaden tres diminutivos pertenecientes al resto del abecedario: plumilla, tejuelo, y zagalejo. Este hecho, que parece contradecir el contenido de los prólogos, podría deberse a esa labor de "retoque" ortográfico de las letras finales a la que los académicos sometieron algunas definiciones. Ciertamente no se trata de nuevas entradas, porque estos términos aparecían ya en el Diccionario, pero al dotarlos de la abreviatura de diminutivo, se alteró sustancialmente la categoría de la palabra.

El prólogo a la tercera edición indica que se han producido nuevos cambios en el cuerpo de la obra, al menos entre las letras $A$ y $F$.

Extendida en el Público y acabada enteramente, procedió la Academia à tercera edición, y en ella ha puesto el Suplemento de la Segunda que fué de las letras $\mathrm{A}, \mathrm{B}$ y $\mathrm{C}$ en sus respectivos lugares alfabéti- 
cos, y siguiendo la idea principiada de ir dando á luz lo que tenga trabajado para la reimpresión de los seis tomos corregidos y aumentados, como se hizo con la letra C, se dan en esta las letras D, E y F, con la corrección y aumento que tiene trabajadas hasta el presente $[\ldots]$.

Por lo que se refiere a las letras siguientes, es decir, de la $G$ en adelante, se repite la idea que aparecía en los prólogos de la primera y de la segunda edición.

La $\mathrm{G}$ y demás letras restantes van sin aumento, ni corrección como están en el DICCIONARIO antiguo; pero alterada la ortografía y conforme a las reglas que ha establecido la ACADEMIA, y que ha observado en las primeras letras que lleva corregidas, pues sería una deformidad muy grande, que en un mismo tomo se usase de diversa ortografía.

De nuevo nos encontramos con que no se ha completado la revisión del Diccionario de Autoridades, pues se incorpora aquí todo el material corregido y aumentado hasta la letra $F$ y se mantienen sin alteración las restantes, salvo en cuestiones de mera ortografía, según se aprecia en el apéndice. Así sucede en la mayor parte de los casos, como se aprecia en el apéndice. Solamente descubrimos una pequeña innovación fuera de los márgenes señalados. Se trata de un diminutivo al que no se alude en el texto preliminar: navajuela, que sí constituye una nueva entrada del diccionario.

3. Si en las tres primeras ediciones, por tanto, el proceso de revisión del Diccionario provocó un número proporcionado de cambios en lo que respecta al número de diminutivos, en la cuarta (1803) el volumen de incorporaciones resulta sorprendentemente elevado, hasta tal punto que supera con creces al de cualquiera de las ediciones precedentes o consecuentes. Se trata de un hecho determinante para la comprensión de la estructura actual de la obra, y su explicación requiere contar tanto con los materiales publicados como con los Libros de Actas y Acuerdos conservados en los archivos de la Real Academia Española.

A pesar de que en 1803 la revisión del Diccionario sólo alcanzaba la 
letra L, la Academia, contra todo pronóstico, había decidido incorporar todas las nuevas voces disponibles. Así lo leemos en el prólogo:

En esta edición, que es la quarta, llega la Academia con el trabajo de su revisión hasta la L inclusive; pero queriendo satisfacer á las insinuaciones de muchas personas, que deseaban se pusiesen las voces que faltaban en las otras letras, ha intercalado en todas ellas cuantas poseia, y quantas han recogido los actuales individuos de la Academia, corrigiendo asimismo algunos artículos de estas combinaciones, añadiendo además varias voces á las letras anteriores, y mejorando las definiciones de otras.

Las listas de diminutivos recogidas en apéndice corroboran estas palabras de la Academia. Evidentemente, se produce una alteración radical del proceso de reforma desarrollado hasta entonces; pero las razones aducidas en el prólogo no son exactas. No se trató de una simple y abstracta satisfacción de "las insinuaciones de muchas personas" a pesar de que es aceptable la existencia de una determinada presión social, ni el peso de su elaboración recayó en el pleno. El motor y protagonista del cambio parece haber sido un pequeño grupo de académicos que trabajaron, eso sí, con el beneplácito del pleno.

La Academia continuaba la revisión del Diccionario, con un trabajo diario de lectura de todas las cédulas de "corrección y aumento" a las que a veces se dedicaba jornadas enteras?. Se trataba de un trabajo lento y arduo que no les permitía entregarse a la nueva edición del diccionario con la atención y celeridad que habría sido deseable. Por ello, el pleno decidió nombrar una comisión de cuatro personas para que, trabajando en juntas ordinarias, publicaran lo más rápidamente posible el volumen esperado. El sistema no era novedoso, como sabemos: se había hecho en la primera edición y, por tanto, se seguía la tradición iniciada entonces por la Academia.

Enterada la Academia del número de Diccionarios pequeños que hay existentes, del tiempo que podrá pasar para concluirse su impre-

7 RAE, Libros de Actas y Acuerdos, 1791-1803. 
sión, y del que es necesario para hacer otra, resolvió que desde luego se continúe en las juntas ordinarias adelantando cuanto se pudiere el trabajo a que se había dado principio para el mejor orden, economía y corrección de la nueva impresión que se ha de hacer [...] nombrando para encargo a los señores Silva, Cabrera, Alamanzón y Valbuena, dándoles al mismo tiempo comisión para que se empiece a imprimir cuando les pareciese conveniente, y los términos que estimen más oportunos ${ }^{8}$.

Las cuatro personas nombradas con este fin eran: Pedro de Silva Meneses y Sarmiento ${ }^{9}$, Ramón Cabrera ${ }^{10}$, Manuel de Valbuena ${ }^{11}$ y Juan Crisóstomo Ramírez Alamanzón ${ }^{12}$, según se deduce del texto transcrito.

Como había sucedido en las ediciones precedentes, se decide incluir en la que se está preparando el conjunto de las cédulas ya revisadas según se lee en los Libros de Actas y Acuerdos. La propuesta correspondía al pleno, pero la ejecución se deja en manos del pequeño grupo delegado. Más adelante tanto la propuesta como la ejecución de otros

8 Ibidem, 3 de febrero de 1795.

9 Pedro de Silva fue nombrado Director de la Academia el 4 de febrero de 1802, y con ese cargo aparece en las listas de miembros del diccionario. También se le presenta como Caballero Comendador de las Elxas en la orden de Alcántara, Bibliotecario mayor de S. M., Consiliario de la Real Academia de S. Fernando y Honorario de la de S. Carlos de Valencia.

10 Ramón Cabrera, Presbítero, Prior de Arroniz, Académico de honor de la Real Academia de S. Fernando, era tesorero de la Academia en 1803. Después de la publicación del diccionario, confeccionó un vocabulario de castellano antiguo que se conserva en la biblioteca de la Real Academia Española.

11 Manuel de Valbuena no aparece como académico de número hasta este momento. En la lista de miembros que se recoge en la cuarta edición del diccionario se le presenta como Regente de Estudios y Director segundo del Real Seminario de Nobles.

12 Juan Crisóstomo Ramírez Alamanzón, aunque previamente fue miembro supernumerario, aparece en el diccionario como académico de número, como Presbítero Académico Supernumerario de la Real Academia de la Historia, como Bibliotecario de la Academia, y como encargado de la impresión del diccionario. Sabemos, gracias a los Libros de Actas y Acuerdos, que fue secretario en algunas ocasiones durante los años en que se estaba elaborando la cuarta edición. 
aspectos relevantes corresponderá a la comisión, quedando el pleno como órgano ratificador.

Habiendo dado cuenta de las varias cédulas de aumento que había para añadirse a las letras que ya estaban corregidas, la Academia, después de haber conferenciado largamente sobre este particular, acordó que se inserten todas en la nueva edición del Diccionario de que se está tratando, siendo antes examinadas y aprobadas en la sala que está destinada para revisión del Diccionario $[\ldots]^{13}$.

En esta situación no sorprende que Pedro de Silva se viera obligado a reclamar más tiempo para que la comisión pudiera dedicarse a sus quehaceres con cierta holgura.

El señor Silva hizo presente que los individuos que entendían en las juntas ordinarias en la nueva revisión del Diccionario, no podían adelantar en ella tanto como deseaban, y pedían las circunstancias por el encargo que se les había hecho posteriormente de examinar las cédulas de aumento que se habían presentado, y que para cumplir con ambas comisiones creía muy oportuno que los cuatro individuos que entienden en ella tuviesen una junta extraordinaria en cada semana. La Academia, conformándose con el parecer de dicho señor, acordó que hubiese una junta extraordinaria cada semana en los términos propuestos y por el tiempo que se creyere necesario para los fines que se han expresado ${ }^{14}$.

Concedida la petición de reunirse en junta extraordinaria cada semana, se plantearon, a partir de ese momento, los asuntos más importantes en el interior de la comisión ${ }^{15} \mathrm{y}$, si ésta lo juzgaba conveniente,

13 RAE, Libros de Actas y Acuerdos, 30 de abril de 1795.

14 Ibidem, 29 de septiembre de 1795.

15 Sólo el prólogo de esta edición no fue obra de la comisión, al menos en su versión definitiva. Estando Alamanzón como secretario, se escribe lo siguiente: "Leí el prólogo que ha de ponerse en el compendio de la quarta edición del Diccionario, y habiéndose ocurrido algunas dudas sobre él, nombró el señor Director a los señores Cabrera, Canseco, Flores, Romanillos y Arnao para que tratasen este asunto entre sí y vean el mejor medio en que se haya de poner en el Diccionario, dando cuenta antes a la Academia de lo que tengan por más conveniente" (RAE, Libros de Actas y Acuerdos, 25 de agosto de 1803). 
se trasladaban al pleno, que comúnmente daba su aprobación. Así sucedió, por ejemplo, con algunas cuestiones ortográficas de relevancia que no se habían tratado con anterioridad.

El señor Silva hizo presente, a nombre de la Junta que está encargada de la revisión y arreglo del Diccionario que se va a imprimir que en el discurso de esta ocupación se había notado que en nuestro Diccionario había muchos nombres primitivos escritos con una ortografía particular que no se observaba en sus derivados y compuestos, pues se veía, por ejemplo: alkali y alkalino escritos con $\mathrm{k}$; y alcalino $\mathrm{y}$ alcalizado con $\mathrm{c}$ [...] Almanak con $\mathrm{k}$ y almanaquero con qu, y que como de esta variedad resultase una incongruencia, que daba enojos, era indispensable que la Academia adoptase una sí otra escritura, para que ésta se siguiese en todos los artículos, y voces que para ello tenían una misma razón y fundamento ${ }^{16}$.

Precisamente entonces la comisión planteó la complicación ortográfica que suponía mantener el grupo culto $p h$ para representar un sonido labiodental fricativo sordo cuando se contaba con una representación más sencilla: $f$. En estos casos, la Academia aprobó "por unanimidad", según se expone en estos documentos, las propuestas de la comisión.

Sobre su papel en la introducción del enorme caudal de diminutivos en todas las letras, hay que tomar como referencia, otra vez, los Libros de Actas y Acuerdos:

Hizo presente el señor Silva, que en las tres ediciones que se han hecho del Diccionario pequeño no se habían añadido otros artículos a la primera que se trabajó por la Academia más que hasta la letra $\mathrm{F}$, con respecto a la revisión de voces de aumento y corrección en que se entendía en todas las juntas: que siendo ésta muy lenta, y notándose una diferencia muy perceptible entre las letras revistas podría examinarse por la junta de Diccionario para la edición que se está tirando un número considerable entre los papeles de la Academia y añadirse aquéllas que estuvieren bien calificadas y probadas, tanto más cuanto que en las últimas letras se echaban de menos muchas

16 Ibidem, 17 de noviembre de 1795. 
voces muy usuales y que era conocido que se habían trabajado con mucho apresuramiento, y habiendo sido esta propuesta muy de la aprobación de la Academia, acordó por uniformidad que así se hiciese $^{17}$.

Del párrafo aquí transcrito se desprenden al menos dos ideas. La primera, de sentido común, nunca se había planteado con tanto rigor: la comisión se percata de que supone un desequilibrio reformar únicamente las letras iniciales del diccionario. La segunda es consecuencia de un criterio pragmático de utilidad: la comisión echa en falta la presencia de voces muy usuales en el diccionario, sobre todo en lo que respecta a las últimas letras, lo que es fundamental para nuestro estudio porque, entre las voces usuales, los diminutivos desempeñan un papel de primer orden.

En todo caso, la postura de la comisión resultaba bastante ambiciosa: pretendía una reforma global y no parcial como la que se había llevado a cabo hasta entonces, y estaba muy decidida a ampliar el caudal léxico. Como consecuencia de la primera idea, la cuarta edición resultó mucho más actualizada que cualquiera de las precedentes, y por la segunda, el nuevo diccionario fue aún más permeable con respecto a la incorporación de diminutivos junto a una gran cantidad de términos nuevos, de forma que es esta cuarta edición una de las más importantes en cuanto a volumen de voces ${ }^{18}$.

\section{BIBLIOGRAFÍA}

Alvar EzQuerra, Manuel (1985): "El diccionario de la Academia a través de sus prólogos: los planteamientos y el vocabulario general", en Philologica hispaniensia in honorem M. Alvar, Madrid, Gredos, pp. 33-44.

PASCUAL, José Antonio (1997): “La caracterización de los arcaísmos en un diccionario de uso", en Lèxic, corpus $i$ diccionaris. Cicle de

17 Ibidem, 28 de junio de 1798.

18 Alvar Ezquerra (1985). 
Conferències 95-96. Barcelona, Institut Universitari de Lingüística aplicada, Universitat Pompeu Fabra.

Real ACAdemia Española (1984): Diccionario de Autoridades, Madrid, Gredos, edición facsímil.

- Diccionario de la Lengua Castellana, ${ }^{19}$ Madrid, $1^{\mathrm{a}}$ ed. $1780,2^{\mathrm{a}}$ ed. $1783,3^{\mathrm{a}}$ ed. $1791,4^{\mathrm{a}}$ ed. $1803,5^{\mathrm{a}}$ ed. $1817,6^{\mathrm{a}}$ ed. $1822,7^{\mathrm{a}}$ ed. 1832 , $8^{\mathrm{a}}$ ed. $1837,9^{\mathrm{a}}$ ed. $1843,10^{\mathrm{a}}$ ed. $1852,11^{\mathrm{a}}$ ed. $1869,12^{\mathrm{a}}$ ed. 1884 , $13^{\mathrm{a}} 1899,14^{\mathrm{a}}$ ed. $1914,15^{\mathrm{a}}$ ed. $1925,16^{\mathrm{a}}$ ed. $1936,17^{\mathrm{a}}$ ed. $1947,18^{\mathrm{a}}$ ed. $1956,19^{\mathrm{a}}$ ed. $1970,20^{\mathrm{a}}$ ed. $1984,21^{\mathrm{a}}$ ed. 1992.

— Libros de Actas y Acuerdos. Archivos de la Real Academia Española.

SECO, Manuel (1991): "Introducción", en Diccionario de la Lengua Castellana reducido a un tomo para su más fácil uso. Edición facsímil. Madrid, pp. III-XII.

19 Desde 1925, la Academia denomina a su obra lexicográfica Diccionario de la Lengua Española. 


\section{APÉNDICE}

Se recogen los diminutivos incorporados al diccionario académico en sus diversas ediciones ${ }^{20}$.

$$
1^{\mathrm{a}} \text { y } 2^{\mathrm{a}}
$$

1. Agrete.

2. Agüita.

3. Almadaneta.

4. Almadraqueja.

4. Amorcillo.

5. Arbolejo.

6. Arenalejo

7. Artalejo.

8. Artalete.

9. Bacinejo.

10. Balconcillo.

11. Baleta.

12. Barbajuelas.

13. Barquete.

14. Barquichuela.

15. Barrilete.

16. Batelejo.

17. Besico.

18. Bestihuela.
19. Bestizuela.

20. Bocelete.

21. Bodegoncillo.

22. Bodegueta.

23. Borrachuelo.

24. Boyezuelo.

25. Bozalejo.

26. Brevete.

27. Bribonzuelo.

28. Caballuelo.

29. Cabezuelo.

30. Calecico.

31. Camellejo.

32. Campaneta.

33. Capillejo.

34. Caracolejo.

35. Castañuelo.

36. Cazoleja.

37. Cedazuelo.
38. Cejuela.

39. Chicorrotico.

40. Chicuelo.

41. Chiquirritico.

42. Chiquirritín

43. Chupeta.

44. Cieguecico.

45. Cieguezuelo.

46. Cofiezuela.

47. Collarejo.

48. Copanete.

49. Copeta.

50. Corezuelo.

51. Cornete.

52. Corpezuelo.

53. Covanillo.

54. Plumilla.

55. Tejuelo.

56. Zagalejo.

$3^{a}$

1. Agracejo.

2. Agujeruelo.

3. Alfanjete.

4. Anqueta.

5. Batatín.

6. Caldereta.

7. Castillete.

8. Chicorrotín.
9. Demoñuelo.

10. Dentecillo.

11. Enagüillas.

12. Enojuelo.

13. Escuderete.

14. Espinilla.

15. Esquileta.

16. Fachuela.
17. Fardelejo.

18. Flequillo.

19. Flotilla.

20. Foguezuelo.

21. Fontezuela.

22. Frontalete.

23. Fueguezuelo.

24. Navajuela.

20 Recogemos todos los sustantivos y adjetivos, pero prescindimos de los adverbios y las interjecciones debido a su escaso número (son cuatro exactamente los que se incorporan al diccionario entre Autoridades y la edición de 1992). 
$4^{a}$

1. Amarillejo.

2. Arcabucete.

3. Atochuela.

4. Babosuelo.

5. Carrascalejo.

6. Cordezuela.

7. Costecilla.

8. Costezuela.

9. Entresuelejo.

10. Escalereja

11. Fortezuelo

12. Fuentezuela.

13. Galeoncete.

14. Gamelleja.

15. Ganchuelo.

16. Garruchuela.

17. Gigantillo.

18. Glosilla.

19. Gordillo.

20. Grajuelo.

21. Grandezuelo.

22. Granito.

23. Guirnaldeta.

24. Gumeneta

25. Hacecillo.

26. Hacendeja.

27. Hacezuelo.

28. Hebilleta.

29. Hebilluela.

30. Hembrilla.

31. Herbecica.

32. Herreruelo.

33. Hierrezuelo.

34. Hilete.

35. Historieta.

36. Hollejuela.

37. Hontanarejo.

38. Hormigüela.

39. Hormiguita.
40. Hortecillo.

41. Hortezuela.

42. Hortezuelo.

43. Hoyito.

44. Huertezuela.

45. Indezuelo.

46. Infantillo.

47. Jarreta.

48. Judihuelo.

49. Latiguillo.

50. Lengüezuela.

51. Librejo.

52. Libretín.

53. Liebrecilla.

54. Liebrezuela.

55. Longuezuelo.

56. Lonjeta.

57. Loseta.

58. Lugarejo.

59. Macetilla.

60. Machuelo.

61. Maderuelo.

62. Madroñuelo.

63. Mancebete.

64. Mandilejo.

65. Manguilla.

66. Manojuelo.

67. Mansito.

68. Mantequilla.

69. Mantilleja.

70. Manzanita.

71. Mañeruelo.

72. Martillejo.

73. Mascareta.

74. Mayorazgüelo

75. Meajuela.

76. Molinejo.

77. Nervezuelo.

78. Odrezuelo.
79. Ojito.

80. Oteruelo.

81. Ovezuelo.

82. Patinillo.

83. Patita.

84. Patrañuela.

85. Pechuelo.

86. Periquillo.

87. Perlezuela.

88. Pernete

89. Picudillo.

90. Pimpollejo.

91. Pinarejo.

92. Placetuela.

93. Playuela.

94. Poblezuelo.

95. Pontecilla.

96. Pontezuelo.

97. Porquecilla.

98. Portecica.

99. Portezuela.

100. Portezuelo.

101. Puertezuelo.

102. Puñalejo.

103. Putuela.

104. Rabillo.

105. Risica.

106. Rubinejo.

107. Rufiancete.

108. Sabandijuela.

109. Saborete.

110. Sacristanejo.

111. Saloncillo.

112. Salseruela.

113. Salvajuelo.

114. Saquete.

115. Sarmentillo.

116. Sarrieta.

117. Sartalejo. 
118. Sarteneja.

119. Senderuelo.

120. Sentenzuela.

121. Serpezuela.

122. Sombrerete.

123. Sonajuela.

124. Sortijuela.

125. Tacita.

126. Tajuelo.

127. Tamborete.

128. Toldillo.

129. Tonelete.

130. Torrecilla.
131. Tortolito.

132. Trabajuelo.

133. Tralleta.

134. Trapillo.

135. Trapito.

136. Trebejuelo.

137. Trochuela.

138. Trompico.

139. Uñeta.

140. Uñuela.

141. Vallejuelo.

142. Vedijuela.

143. Vejecito.
144. Vejete.

145. Vejezuelo

146. Ventecico.

147. Ventrecillo.

148. Ventrezuelo.

149. Versecillo.

150. Versete.

151. Viejezuelo.

152. Vinillo.

153. Viñuela.

154. Volcanejo.

$5^{a}$

1. Atabalete

2. Bajete.

3. Bajuelo.

4. Cajeta.

5. Cajetín.

6. Cajuela.

7. Cojinete.

8. Cojuelo.

9. Corpiño.

10. Costanilla.

11. Cuchilleja.

$6^{\mathrm{a}}-11^{\mathrm{a}}$

1. Alegrete.

2. Cachigordete.

3. Chiquitín.

4. Cornetín.

5. Ensaladilla.

6. Espartillo.

7. Fajilla.

8. Febrerillo.

9. Folletín.

10. Frasquete.

11. Fueguecito.
12. Escarabajuelo.

13. Facilillo.

14. Fajuela.

15. Frailecito.

16. Gachuela.

17. Higuerilla.

18. Higuillo.

19. Jergueta.

20. Orejeta.

21. Orzuela.

22. Pajarito.

12. Gacheta.

13. Gorreta

14. Grosezuelo.

15. Habladorzuelo

16. Hilanderuelo.

17. Judihuela.

18. Lentejuela.

19. Mayorazguete.

20. Meloncillo.

21. Menorete.

22. Merendilla.
23. Penachuelo.

24. Pendenzuela.

25. Realejo.

26. Rosita.

27. Tijereta.

28. Tijerilla.

29. Tijeruela.

30. Trastuelo.

31. Vejigüela.
23. Monterilla.

24. Monuelo.

25. Morterete.

26. Mujerzuela.

27. Muñequilla.

28. Nadilla.

29. Nadita.

30. Navichuela.

31. Nerviecillo.

32. Peñuela.

33. Picardihuela. 
34. Plazoleta.

35. Pobrete.

36. Rodezuela.
37. Tineta.

38. Tontuelo.

39. Tunantuelo.
40. Vejiguilla.

41. Locuelo.

42. Maceta.

43. Malejo.

44. Maletín.

45. Manjarejo.

46. Mocosuelo.

47. Mollejuela.

48. Naveta.

49. Ojete.

50. Palanqueta.

51. Pandereta.

52. Pañete.

53. Patiecillo.

54. Pesebrejo.

55. Pilluelo.

56. Pilotín.

57. Serratilla.

58. Serreta.

59. Tamborilete.

60. Zarzuela.

25. Jovenzuelo.

26. Jueguezuelo.

27. Lagartijo.

28. Lanchuela.

29. Lentezuela.

30. Lloramico.

31. Macillo.

32. Mallete.

33. Manjarete.

34. Mantehuelo.

35. Matorralejo.

36. Mazuelo. 
37. Mesita.

38. Mirilla.

39. Monjita.

40. Murete.

41. Necezuelo.

42. Orejuela.

43. Pedreta.

44. Pillete.

45. Pillín.

46. Poyete.

47. Realete.

48. Romanillo.

49. Roqueta.

$16^{\mathrm{a}}-21^{\mathrm{a}}$

1. Asuntejo.

2. Azuquita.

3. Azuquítar.

4. Bellotillo.

5. Berenjenín.

6. Bosquete.

7. Caminejo.

8. Canillita.

9. Casuca.

10. Chatungo.

11. Chichito.

12. Chiquilín.

13. Cogotillo.

14. Contezuelo.

15. Culillo.

16. Flechilla.

17. Gallinita.

18. Ganchete.

19. Gazpachuelo.

20. Gigantilla.
50. Rosillo.

51. Rostrillo.

52. Sacadilla.

53. Saetín.

54. Saladillo.

55. Sayuela.

56. Segundillo.

57. Serija.

58. Silletín.

59. Sonetillo.

60. Tabloncillo.

61. Tochuelo.

62. Todito.

21. Gordezuelo.

22. Gozquillo.

23. Gradecilla.

24. Hierbezuela.

25. Hijuco.

26. Infantejo.

27. Jarrita.

28. Levitín.

29. Machito.

30. Mamaíta.

31. Manita.

32. Mañanica.

33. Menoreta.

34. Mesita.

35. Miseriuca.

36. Mismito.

37. Monín.

38. Mundillo.

39. Negrito.

40. Nietezuelo.
63. Toldilla.

64. Tomatillo.

65. Tonillo.

66. Torcidillo.

67. Torito.

68. Triguillo.

69. Triquete.

70. Truchuela.

71. Tunantuela.

72. Verdejo.

73. Verdezuelo.

74. Zarzaganillo.

75. Zumillo.

41. Nuececilla.

42. Palabreja.

43. Palmita.

44. Pañolito.

45. Papaíto.

46. Pequeñín.

47. Pernezuela.

48. Portichuelo.

49. Potrillo.

50. Ramalillo.

51. Rejín.

52. Retamilla.

53. Sobaquillo.

54. Sortija.

55. Tenedorcillo.

56. Ternez.

57. Tito.

58. Vejeta.

59. Zoleta 